\title{
Molecular Mechanism of Water Reorientation Dynamics in Dimethyl Sulfoxide
}

\section{Aqueous Mixtures}

Xia Zhang ${ }^{1}$, Zhangtao Wang ${ }^{3}$, Zhening $\mathrm{Chen}^{2}$, Hui $\mathrm{Li}^{3}$, Lu Zhang ${ }^{2}$, Jinting Ye ${ }^{1}$, Qiang Zhang ${ }^{*}$, Wei Zhuang ${ }^{2^{*}}$

1 College of Chemistry and Materials Science, Inner Mongolia University for Nationlities, Tongliao, Inner Mongolia 028043, China

2 State Key Laboratory of Structural Chemistry, Fujian Institute of Research on the Structure of Matter, Chinese Academy of Sciences, Fuzhou, Fujian 35000

3 College of Chemistry and Chemical Engineering, Bohai University, Jinzhou, Liaoning 121013, China

* To whom correspondence should be addressed. E-mail: zhangqiang@bhu.edu.cn; wzhuang@f,jirsm.ac.cn

A. Computation of the reorientation times using Extended Molecular Jump model: The reorientation time of water molecule can be obtained from a second-order Legendre polynomial of the reorientation correlation function of $\mathrm{OH}$ bond of water. ${ }^{1-6}$

$\mathrm{C}_{2}(\mathrm{t})=\frac{1}{2}\left\langle 3\left[\widetilde{\mathbf{u}}_{\mathrm{OH}}(\mathrm{t}) \cdot \widetilde{\mathbf{u}}_{\mathrm{OH}}(0)\right]^{2}-1\right\rangle$

$\widetilde{\mathbf{u}}_{\mathrm{OH}}$ is the $\mathrm{OH}$ bond vector of water molecule to be measured. $\mathrm{C}_{2}(\mathrm{t})$ is usually employed to obtain the reorientation time by fitting the following function within $0-10$ ps as previous works. ${ }^{3-5}$

$\mathrm{C}_{2}(\mathrm{t})=\left[(1-\mathrm{A}) \exp \left(-\mathrm{t} / \tau_{1}\right)+\mathrm{A}\right] \exp \left(-\mathrm{t} / \tau_{2}\right)$

$\mathrm{A}$ is a prefactor of the exponential functions. $\tau_{1}$ is the librational relaxation time. $\tau_{2}$ is the reorientation times related to the long time reorientation times directly from the molecular dynamic simulations.

The Extended Molecular Jump model ${ }^{2}$ (EMJ) was used here to calculate the water reorientation time around $\mathrm{OH}$ bond vector, which occurs as a large amplitude angular jump of water $\mathrm{OH}$ bond from one hydrogen bond acceptor to another. In pure water, the reorientation correlation function of water (Eq. S1) is considered as the product of two independent correlation functions: a jump reorientation of $\mathrm{OH}$ bond vector within the local hydrogen bond frame $\mathrm{C}_{\mathrm{J}}(t)$ and a correlation function of the intact 
hydrogen bond frame $\mathrm{OO}$ vector (the frame reorientation) $\mathrm{C}_{\mathrm{f}}(\mathrm{t})$ during hydrogen bond switching. ${ }^{2-7}$

$$
\mathrm{C}_{2}(\mathrm{t})=\left\langle\mathrm{P}_{2}\left[\widetilde{\mathbf{u}}_{\mathrm{OH}}(0) \widetilde{\mathbf{u}}_{\mathrm{OH}}(\mathrm{t})\right]\right\rangle=\left\langle\mathrm{P}_{2}\left[\widetilde{\mathbf{u}}_{\mathrm{H}}(0) \widetilde{\mathbf{u}}_{\mathrm{H}}(\mathrm{t})\right]\right\rangle \times\left\langle\mathrm{P}_{2}\left[\widetilde{\mathbf{u}}_{\mathrm{OO}}(0) \widetilde{\mathbf{u}}_{\mathrm{OO}}(\mathrm{t})\right]\right\rangle=\mathrm{C}_{\mathrm{J}}(\mathrm{t}) \mathrm{C}_{\mathrm{f}}(\mathrm{t})
$$

here $\widetilde{\mathbf{u}}_{\mathrm{H}}$ is the unit vector of water $\mathrm{OH}$ bond within the local hydrogen bond OO frame and $\widetilde{\mathbf{u}}_{\mathrm{OO}}$ is the orientation vector of hydrogen bond frame $\mathrm{OO} . \mathrm{O}$ is one hydrogen bond acceptor, referring to a water oxygen atom in pure water.

In DMSO-water mixtures, there are two types of hydrogen bond acceptor: the oxygen atom of water and the oxygen atom of DMSO. Therefore, we can group the simulated hydrogen bond switching events into four types, by first dissecting the trajectories of each donor water molecule into a group of successive events. Each event begins when the previous switching finishes and the donor water molecule forms hydrogen bond with the current acceptor, and it ends at the moment that the donor forms the hydrogen bond with the next acceptor. We therefore categorize these events into four groups according to the types of initial and final hydrogen bond acceptors (i->j): from water to water (W->W), from water to DMSO (W->D), from DMSO to water (D->W) and from DMSO to DMSO (D->D).

In the DMSO-water mixtures, the total correlation function of water Eq. S3 can further be decomposed into four independent correlation functions corresponding to four types of hydrogen bond switchings. ${ }^{3-4}$

$\mathrm{C}_{2}(\mathrm{t})=\mathrm{A}_{\mathrm{W} \rightarrow \mathrm{W}}\left\langle\mathrm{P}_{2}\left[\overrightarrow{\mathrm{u}}_{\mathrm{OH}}(0) \overrightarrow{\mathrm{u}}_{\mathrm{OH}}(\mathrm{t})\right]\right\rangle_{\mathrm{W} \rightarrow W}+\mathrm{A}_{\mathrm{W} \rightarrow \mathrm{D}}\left\langle\mathrm{P}_{2}\left[\overrightarrow{\mathrm{u}}_{\mathrm{OH}}(0) \overrightarrow{\mathrm{u}}_{\mathrm{OH}}(\mathrm{t})\right]\right\rangle_{\mathrm{W} \rightarrow \mathrm{D}}+$

$\mathrm{A}_{\mathrm{D} \rightarrow \mathrm{W}}\left\langle\mathrm{P}_{2}\left[\overrightarrow{\mathrm{u}}_{\mathrm{OH}}(0) \overrightarrow{\mathrm{u}}_{\mathrm{OH}}(\mathrm{t})\right]\right\rangle_{\mathrm{D}->W}+\mathrm{A}_{\mathrm{D} \rightarrow \mathrm{D}}\left\langle\mathrm{P}_{2}\left[\overrightarrow{\mathrm{u}}_{\mathrm{OH}}(0) \overrightarrow{\mathrm{u}}_{\mathrm{OH}}(\mathrm{t})\right]\right\rangle_{\mathrm{D} \rightarrow \mathrm{D}}$

$\left\langle\mathrm{P}_{2}\left[\overrightarrow{\mathrm{u}}_{\mathrm{OH}}(0) \overrightarrow{\mathrm{u}}_{\mathrm{OH}}(\mathrm{t})\right]\right\rangle_{\mathrm{i} \rightarrow>\mathrm{j}}$ is a second-order Legendre polynomial of the reorientation correlation function of $\mathrm{OH}$ bond of water forming hydrogen bond with acceptor $\mathrm{i}$, and switching its hydrogen bond to acceptor $\mathrm{j}$ subsequently. $\mathrm{A}_{\mathrm{i}->\mathrm{j}}$ is the weighting factor for a type of hydrogen bond switch from acceptor $\mathrm{i}$ to acceptor $\mathrm{j}$, which is calculated as the probability of a water molecule to currently form hydrogen bond with acceptor type $\mathrm{i}$ and next form hydrogen bond with acceptor type $\mathrm{j}$ during the subsequent hydrogen bond switching. $\sum \mathrm{A}_{\mathrm{i}->\mathrm{j}}=1$. According to the EMJ model, the total reorientation correlation function of water along the $\mathrm{OH}$ bond vector therefore is equivalent to the summation of four types of hydrogen bond switchings as equation S5a, if each sub-process in Eq. S4 takes the form of single exponential decay.

$$
\mathrm{C}_{2}(\mathrm{t})=\sum \mathrm{A}_{\mathrm{i} \rightarrow \mathrm{j}} \mathrm{e}^{-\mathrm{t} / \tau_{\mathrm{EMJ}}^{\mathrm{i}} \vec{j}^{j}}
$$


$\tau_{\mathrm{EMJ}}^{\mathrm{i}-{ }^{j}}=1 / \tau_{\mathrm{J}}^{\mathrm{i}->j}+1 / \tau_{\mathrm{f}}^{\mathrm{i}->j}$

(S5b)

The reorientation time of each hydrogen bond switching type $\mathrm{i}->j, \quad \tau_{\mathrm{EMJ}}^{\mathrm{i}} \vec{j}^{j}$ further can be decomposed into summation of its jump and frame components, $\tau_{\mathrm{J}}^{\mathrm{i}->j}$ and $\tau_{\mathrm{f}}^{\mathrm{i}->j}$ in S5b and shown in Fig. 3 in main text. Then the total reorientation time of water, $\tau_{\mathrm{EJM}}$, is a summation of the contributions from four hydrogen bond switchings (Eq. S6a). The total jump reorientation time and the total frame reorientation of water in the mixtures, $\tau_{J}$ and $\tau_{f}$, are approximately defined as equations S6b and S6c. They are used to describe the total contributions of the jump and the frame reorientation of water to $\tau_{\mathrm{EJM}}$ shown in Fig. 1 of main text.

$$
\begin{aligned}
& \tau_{\text {EJM }}=\sum \mathrm{A}_{\mathrm{i} \rightarrow>\mathrm{j}} \tau_{\mathrm{EMJ}}^{\mathrm{i}->j} \\
& \tau_{\mathrm{J}}=\sum \mathrm{A}_{\mathrm{i} \rightarrow>\mathrm{j}} \tau_{\mathrm{J}}^{\mathrm{i}->j} \\
& \tau_{\mathrm{f}}=\sum \mathrm{A}_{\mathrm{i} \rightarrow>\mathrm{j}} \tau_{\mathrm{f}}^{\mathrm{i}->j}
\end{aligned}
$$

Note that when the frame becomes predominant, these prefactors can therefore still capture the frame contribution since the jump and frame contributions share the same weight for each type of hydrogen bond switching.

According to the EMJ model, the frame reorientation time $\tau_{\mathrm{f}}^{\mathrm{i}->j}$ is obtained by fitting the frame correlation function at second order with a single exponential function, ${ }^{2-5}$ while the jump reorientation times $\tau_{\jmath}^{\mathrm{i}->j}$ is calculated by the numerical summation of Eq. S7, given a unsymmetrical distribution of the reorientation jump angle, ,-5 $^{3-5}$

$$
\tau_{\mathrm{J}}^{\mathrm{i}->j}=\tau_{\mathrm{HB}}^{\mathrm{i}->j} \mathrm{~F}\left(\theta^{\mathrm{i}->j}\right)=\tau_{\mathrm{HB}}^{\mathrm{i}->j} \sum \mathrm{P}\left(\theta^{\mathrm{i}->j}\right) \mathrm{f}\left(\theta^{\mathrm{i}->j}\right)
$$

$\tau_{\mathrm{HB}}^{\mathrm{i}->j}$ is the hydrogen bond lifetime of hdyrogen bond switching $\mathrm{i}->\mathrm{j} . \mathrm{P}\left(\theta^{\mathrm{i}->j}\right)$ is the population of the hydrogen bond switching from $i$ to $j$ with the jump angle $\theta^{i->j} . f\left(\theta^{i->j}\right)$ is a function of jump angle according to the Ivanov jump model. ${ }^{6}$

$$
\mathrm{f}\left(\theta^{\mathrm{i}>\mathrm{j}}\right)=\left\{1-\frac{1}{5} \frac{\sin (5 / 2) \theta^{\mathrm{i}->\mathrm{j}}}{\sin \left(\theta^{\mathrm{i}>\mathrm{j}} / 2\right)}\right\}^{-1}
$$

$\theta^{i->j}$ is defined as the angle XOY (O: donor water oxygen, X: the old hydrogen acceptor i, Y: the new hydrogen bond acceptor $\mathrm{j}$ ) for hydrogen bond switching $\mathrm{i}->\mathrm{j}$ at the transition state. It is an intrinsic variable depending on the types of $\mathrm{i}$ and $\mathrm{j}$ during the hydrogen bond switching and is extracted from 3 molecular dynamic trajectories by searching all of the hydrogen bond switching vents. 
The hydrogen bond lifetime $\tau_{\mathrm{HB}}^{\mathrm{i}-{ }^{>} j}$ in Eq. S7 is obtained by fitting the complementary hydrogen bond correlation function $1-\mathrm{C}_{\mathrm{HB}}^{\mathrm{i}}{ }^{>j}(\mathrm{t})$ according to the Stable States Pictures (SSP). ${ }^{2}$ The hydrogen bond switching starts at the moment that $\mathrm{H}^{*}$ hydrogen bonds to $\mathrm{i}$ (water, DMSO), and ends at the moment that $\mathrm{H}^{*}$ hydrogen bonds to a new partner $\mathrm{j}$. The hydrogen bond correlation function $\mathrm{C}_{\mathrm{HB}}^{\mathrm{i}->j}(\mathrm{t})$ presents a probability of forming a stable product at time $\mathrm{t}$, when the system is in the reactant state at $\mathrm{t}=$ 0 is:

$\mathrm{C}_{\mathrm{HB}}^{\mathrm{i}-{ }^{\prime}}(\mathrm{t})=\left\langle\mathrm{n}_{\mathrm{i}}^{\mathrm{R}}(0) \mathrm{n}_{\mathrm{j}}^{\mathrm{P}}(\mathrm{t})\right\rangle$

$\mathrm{n}_{\mathrm{i}}^{\mathrm{R}}(0)$ and $\mathrm{n}_{\mathrm{j}}^{\mathrm{P}}(\mathrm{t})$ are the probability for the system to be in the stable reactant and product states. $\tau_{\mathrm{HB}}^{\mathrm{i}->j}$

(t) is derived from its complementary correlation function at time range of $0 \sim 10 \mathrm{ps}$.

$$
\left(1-\mathrm{C}_{\mathrm{HB}}^{\mathrm{i}->\mathrm{j}}(\mathrm{t})\right)=\exp \left(-\mathrm{t} / \tau_{\mathrm{HB}}^{\mathrm{i}>\mathrm{j}}(\mathrm{t})\right)
$$

The strict hydrogen bond criteria is used for $\mathrm{C}_{\mathrm{HB}}^{\mathrm{i}-j}(\mathrm{t})$, so that the hydrogen bond switching events really happened and excludes the transient breaking of hydrogen pair. Two molecules are considered to be hydrogen bonded if $\mathrm{R}_{\mathrm{OX}}<3.1 \AA, \mathrm{R}_{\mathrm{HX}}<2.0 \AA$ and the hydrogen bond angle $\theta_{\mathrm{HXY}}<20^{\circ}$ according to the pair radial distribution functions of water-water and water-DMSO. X denotes the hydrogen bond acceptor, here oxygen atom of water or DMSO.

To outline the numerical procedure of the EMJ model in this work, (1) we search the hydrogen bond switching events for each water hydrogen atom from old acceptor $\mathrm{i}$ to new acceptor $\mathrm{j}(\mathrm{i}->j$ ) defined in Eq. S4 along the trajectory and get the lifetime of hydrogen bond by fitting the complementary function of Eq. S10. (2) The jump reorientation time of water for each type of hydrogen bond is derived according to Eq. S7 with the jump angle distributions at the transition state of each hydrogen bond switching. (3) We calculate the frame reorientation correlation $\left(\mathrm{C}_{\mathrm{f}}(\mathrm{t})\right)$ function of each hydrogen bond switching in Eq. S3 and S4 and get the frame reorientation time by fitting the frame reorientation correlation function with single exponential function. (4) The reorientation time of water along $\mathrm{OH}$ bond vector for each hydrogen bond switching is obtained with the equation form of Eq. S5b for each hydrogen bond switching. (5) At last, the reorientation time of water $\left(\tau_{\mathrm{EMJ}}\right)$ is calculated by a weighted summation of four hydrogen bond switches with Eq. S6a.

To quantitatively demonstrate how the hydrogen bond strength influences its lifetime, we also calculated the free energy of hydrogen bond according to Eq.4 in method section. $\Delta \mathrm{G}$ is the reversible work $\mathrm{W}(\mathrm{r}, \beta)$ required to bring hydrogen bond donor and acceptor molecules from infinite separation 
113 and random orientation to a hydrogen bond state with a distance $r$ and angle $\beta$ (see the definitions of 114 them in Fig.S7). $\Delta \mathrm{G}$ of the water-water and water-DMSO hydrogen bonds both decrease monotonously 115 with similar magnitude as DMSO mole fraction (Fig.4B) increments before $x_{\mathrm{D}}=0.5$. As $x_{\mathrm{D}}$ changes 116 from 0 to $\sim 0.5$, free energy of both types of hydrogen bond decreases for $\sim 0.7 \mathrm{kcal} / \mathrm{mol}$. At the same 117 time, free energy of DMSO-water hydrogen bond is lower than that of water-water hydrogen bond. The 118 free energy difference between both types is $\sim 0.3 \mathrm{kcal} / \mathrm{mol}$. When $x_{\mathrm{D}}>0.5$, free energy curve of 119 water-water hydrogen bond gradually converges into a plateau, while that of DMSO-water continues to 120 decrease $\left(\sim 0.25 \mathrm{kcal} / \mathrm{mol}\right.$ from $x_{\mathrm{D}}=0.5$ to $\left.x_{\mathrm{D}}=0.9\right)$. The free energy difference between both types 121 becomes as large as $\sim 0.6 \mathrm{kcal} / \mathrm{mol}$ at $x_{\mathrm{D}}=0.9$.

122 The accessibility of potential acceptor (steric effect $)^{2 \mathrm{c}}$ can be estimated by the density $\rho(\mathbf{r})$ of the potential acceptors $\mathrm{O}^{\mathrm{b}}$ (the oxygen atoms of water or DMSO) around the initial acceptor $\mathrm{O}^{\mathrm{a}}$ (Fig.5C-F).

124 Integrating the first peak of the density profile of potential acceptor $\rho(\mathbf{r})$ gets the number $N$ of the new potential hydrogen bond acceptor around the initial hydrogen bond acceptor (Fig.5A-B). For D->W and D->D switches, the first peak in density of water oxygen $\rho_{\mathrm{W}-\mathrm{D}}^{\mathrm{W}}$ around oxygen of initial acceptor DMSO monotonously decreases with increasing DMSO mole fraction, on the other hand there is almost no DMSO oxygen next to the initial acceptor oxygen due to the hydrophobic aggregates. The total number of the potential acceptor for D->W and D->D therefore continuously decreases (Fig. 5A-B), which lead to the monotonously slowing down jumps in Fig.2A. For W->W and W->D switches, the density of water oxygen around initial acceptor water oxygen $\rho \mathrm{W}-\mathrm{W}$ decreases monotonously, while the density of DMSO oxygen, $\rho_{\mathrm{W}}^{\mathrm{D}}-\mathrm{W}$, increases. As a result, the total number of the potential acceptor gradually converges at higher DMSO fraction (Fig.5A), which leads to the observed plateau in their

\section{B. Coarse Graining Jump model}

According to the Eyring model, ${ }^{8}$ the structural relaxation rate is proportional to $\tau_{\mathrm{HOP}} / \mathrm{L}_{\mathrm{LOP}}^{2}$, where

$138 \tau_{\mathrm{HOP}}$ and $\mathrm{L}_{\mathrm{HOP}}$ are the time and length scales of the "excited hoppings" of molecules or collections of 139 molecules from one "local basin" to another. ${ }^{9}$ Intermittently the water molecule hops from one 140 dynamical basin to another. The position of the center of mass of a chosen water molecule during 500ps 141 at $x_{\mathrm{D}} \approx 0.66$. The trajectory shows a conspicuous aggregation with well-defined dynamical basins 
142 (represented schematically in different colors). We can apply a coarse graining time algorithm to the 143 single-molecule water trajectories to quantitatively rationalize the magnitude and concentration 144 dependences of the hopping time $\tau_{\mathrm{HOP}}$ and the jump length $\mathrm{L}_{\mathrm{HOP}}$.

145 In this algorithm, the center of the $k^{\prime}$ th dynamic basin of the tagged water molecule, $\overline{\mathrm{R}}(\mathrm{n})_{\mathrm{k}}$, is the 146 running average of the molecular center-of-mass $\bar{R}(n)$ (Eq. S11): ${ }^{9}$

$147 \quad \bar{R}(n)_{k}=\frac{(n-1) \bar{R}(n-1)_{k}+R(n)}{n}$

148 The average is updated until $\bar{R}(n+1)$ walks out of a certain range:

$\left|R(n+1)-\bar{R}(n)_{k}\right|\left\{\begin{array}{lc}\leq D_{\max } & \text { update the basin center position } \\ >D_{\max } & \text { start the new basin }\end{array}\right.$

150 We set $D_{\max }=1.5 \AA,{ }^{9}$ which was found to be a reasonable value for the pure water, as a threshold of the 151 basin-crossing. The past $n$ snapshots are defined as a dynamical basin centered at $\overline{\mathrm{R}}(\mathrm{n})_{\mathrm{k}}$. If the labeled 152 molecule hops into a new basin according to Eq. S12, the running index $n$ is updated to 1 . The hopping 153 time $\tau_{\text {HOP }}$ is defined as the average waiting time of water within dynamic basin $\bar{R}(n)_{k}$, while the jump 154 length $\mathrm{L}_{\mathrm{HOP}}$ is the average distance between two consecutive basin centers, $\left\langle\overline{\mathrm{R}}_{\mathrm{k}+1}-\overline{\mathrm{R}}_{\mathrm{k}}\right\rangle$.

156 Pair correlation function: The pair correlation functions, $\mathrm{R}(\mathrm{t})$ represents the probability that one pair 157 exists at $t=0$ is still intact at a later time $t$,

$158 \quad \mathrm{R}(\mathrm{t})=\langle\mathrm{h}(\mathrm{t}) \mathrm{h}(0)\rangle /\langle\mathrm{h}(0)\rangle$

$159 \mathrm{~h}(\mathrm{t})$ is the population operator. $\mathrm{h}(\mathrm{t})=1$ when two molecules are within first solvation shell of each other, 160 and $\mathrm{h}(\mathrm{t})=0$ otherwise. The angular brackets denote the ensemble average. 


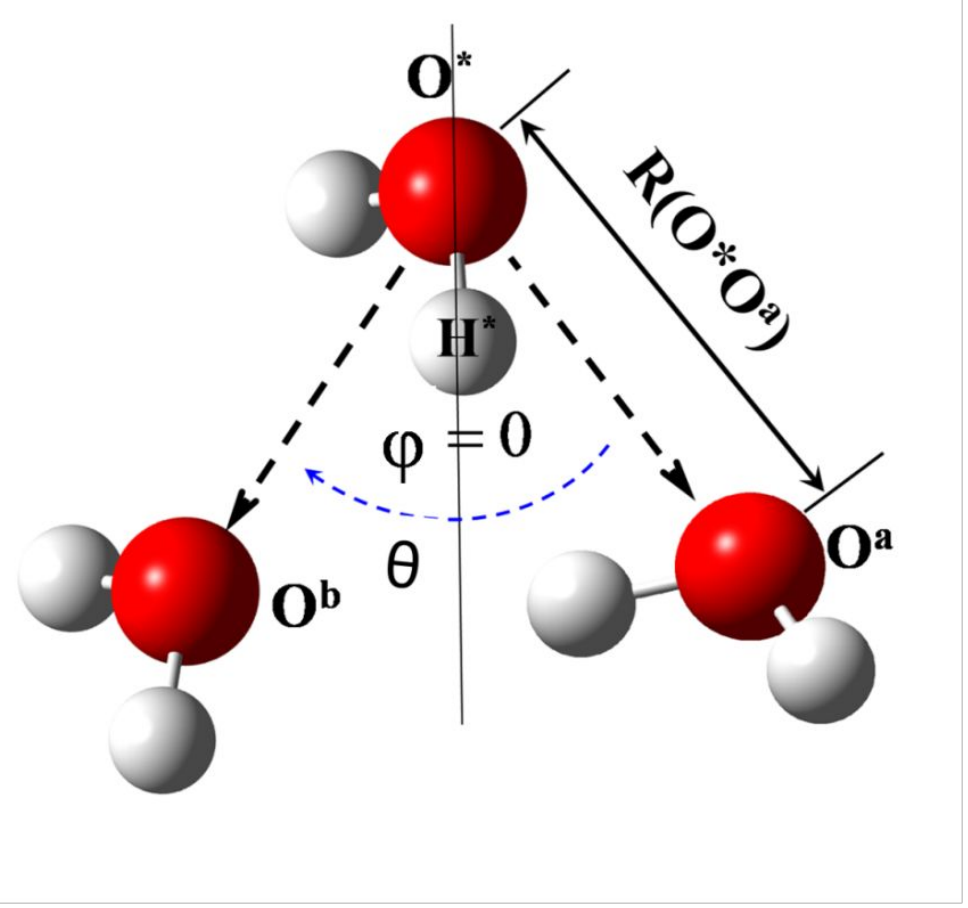

163 Fig. S1 The definitions of the reaction variables along the $\mathrm{W}->\mathrm{W}$ hydrogen bond switching path from initial acceptor $\left(\mathrm{O}^{\mathrm{a}}\right)$ to final acceptor $\left(\mathrm{O}^{\mathrm{b}}\right): \varphi$ is the angle between the $\mathrm{O}^{*} \mathrm{H}^{*}$ vector and the bisector plane of $\mathrm{O}^{\mathrm{a}} \mathrm{O}^{*} \mathrm{O}^{\mathrm{b}}, \varphi \approx-35$ before the hydrogen bond switching. $\theta$ is the angle between the vector $\mathrm{O}^{*} \mathrm{O}^{\mathrm{a}}$ and vector $\mathrm{O}^{*} \mathrm{O}^{\mathrm{b}}$ at the transition state of hydrogen bond switching, which is considered as the jump angle approximately. $\mathrm{R}_{\mathrm{O}^{*} \mathrm{O}} \mathrm{a}, \mathrm{R}_{\mathrm{O}^{*} \mathrm{O}} \mathrm{b}$ and $\mathrm{R}_{\mathrm{O}}{ }_{\mathrm{O}} \mathrm{b}$ are the distances of the donor oxygen and the initial acceptor oxygen, the distances of the donor oxygen and thefinal acceptor oxygen and the distances of the initial acceptor oxygen and the final acceptor oxygen. 

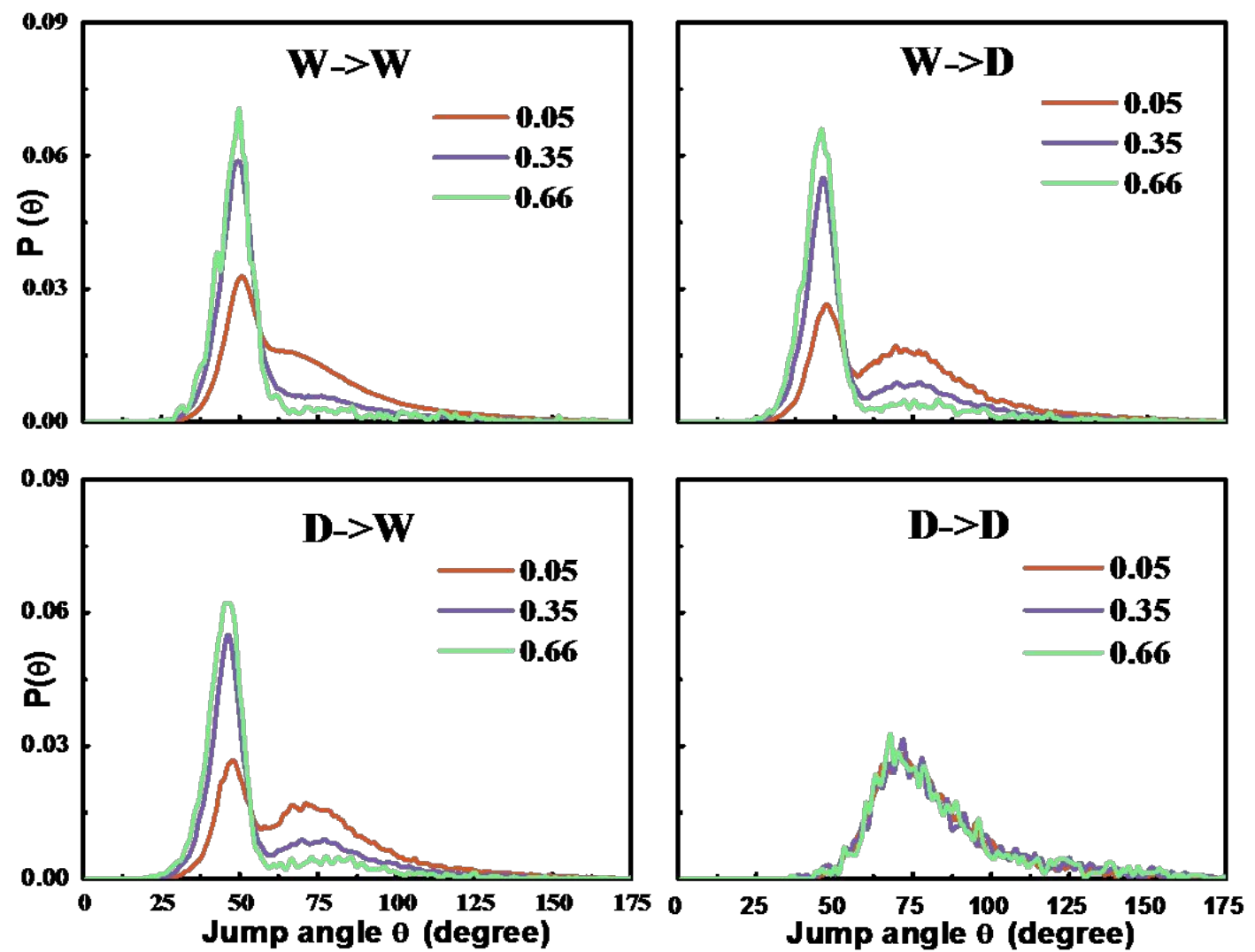

Fig. S2 The distributions of the jump angle for the four hydrogen bond switching types: (1) water-to-water (W->W); (2) water-to-DMSO (W->D); (3) DMSO-to-water (D->W) and (4) DMSO-to-DMSO (D->D).

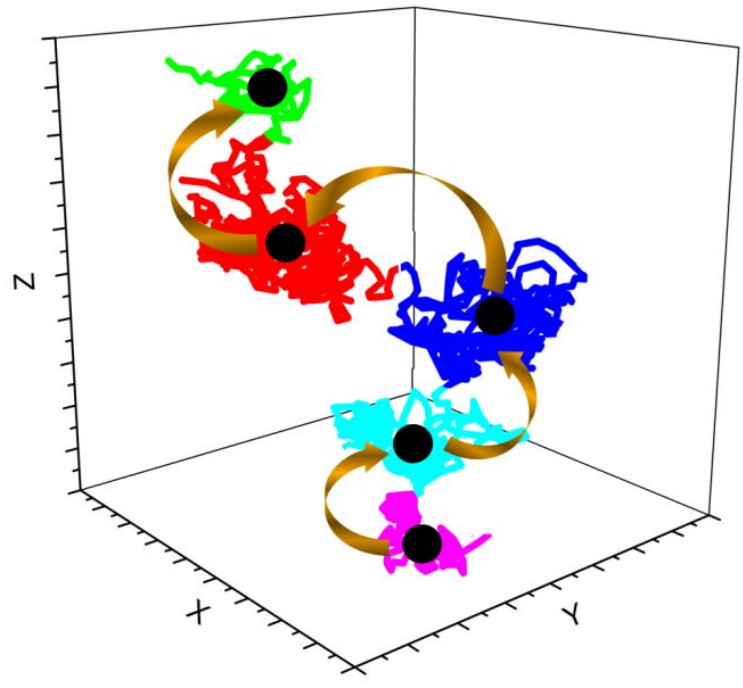

Fig. S3 The position of the center of mass of a chosen water molecule during 500ps at $x_{\mathrm{D}} \approx 0.66$. The trajectory shows a conspicuous aggregation with well-defined dynamical basins (represented schematically in different colors). 

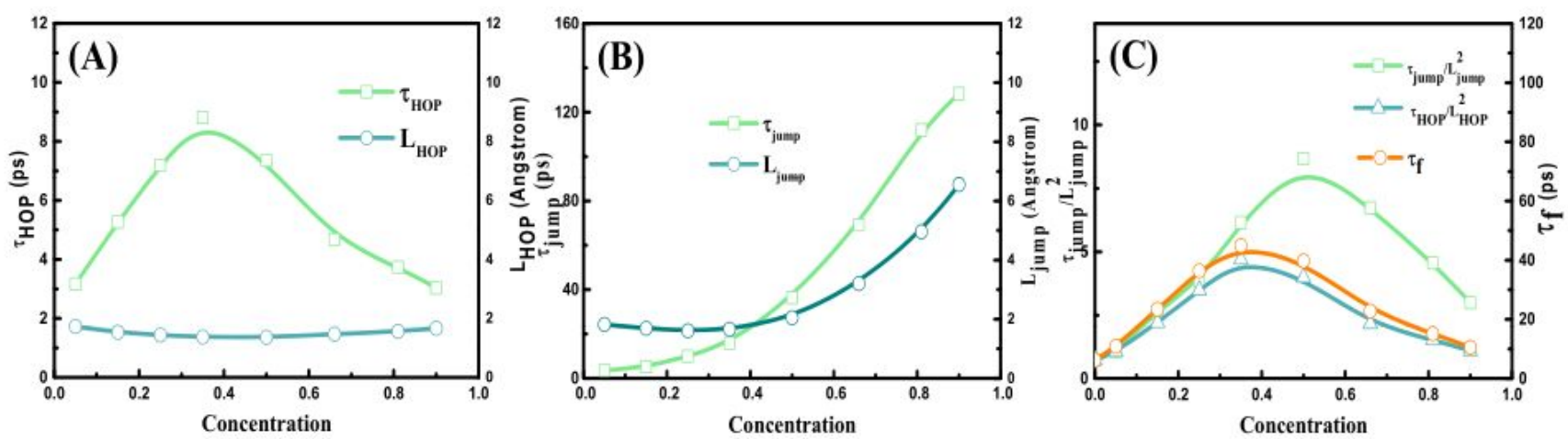

Fig. S4 (A) the hopping time $\tau_{\mathrm{HOP}}$ (ps) and the hopping length (angstrom) $\mathrm{L}_{\mathrm{HOP}}$ with a continuous time random walk

(ps) (the average interval between any two consecutive hydrogen bond switchings) and length (angstrom) $\mathrm{L}_{j u m p}$ (the distance between the average positions 182 of water molecule hydrogen bonding to the old and the new hydrogen bond partners before and after hydrogen bond 183 switching), (C) the correlation plots of $\tau_{\mathrm{jump}} / \mathrm{L}_{\mathrm{L}}^{2}, \tau_{\mathrm{HOP}} / \mathrm{L}_{\mathrm{LOP}}^{2}$ (defined as Eq. S10) and the frame reorientation time of 184 water, $\tau_{\mathrm{f}}$

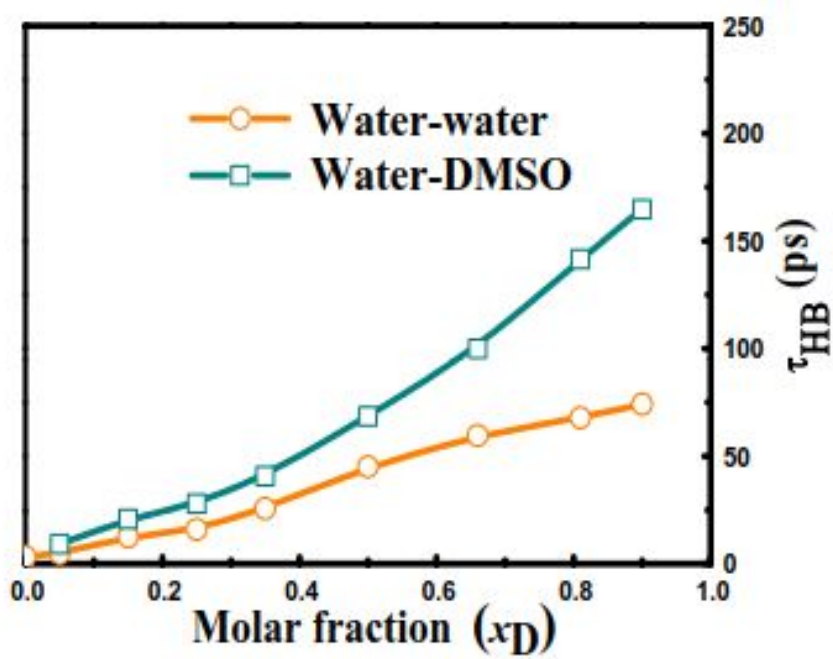

Fig. S5 The lifetimes of water-water and water-DMSO hydrogen bonds at different DMSO mole fractions. 


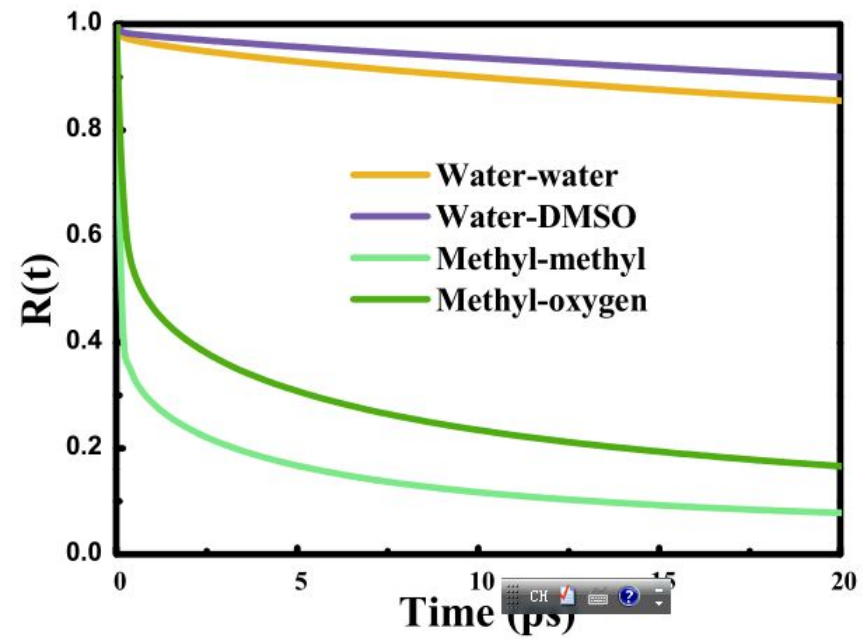

189 Fig. S6 R(t), the pair correlation functions of the water-water, water-DMSO, methyl-methyl and the methyl-oxygen 190 pairs (Eq.S13). The integration of the pair correlation function is the average residence time of one molecule 191 staying within the first solvation shell of its partner.

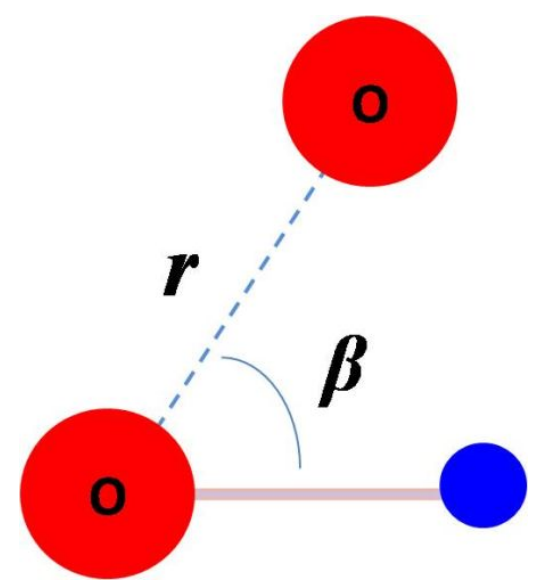

193 Fig. S7 The definition of the variables, $r$ and $\beta$ of the potential of mean force $W(r, \beta)$ for the free energy of hydrogen bond.

Table S1 the dynamic properties of water and DMSO in the DMSO-water mixtures, including the diffusion constants and the reorientation times of water and DMSO, the reorientation time of DMSO with different numbers of hydrogen bonds, the hydrogen bond lifetimes, average jump angles, the jump reorientation time, the frame reorientation time of 199 each hydrogen bond switching, as well as the ratio of $\tau_{\mathrm{HB}}^{W->D} / \tau_{\mathrm{HB}}^{\mathrm{D}} \rightarrow W$, the equilibration constants $\mathrm{K}=\mathrm{A}_{\mathrm{W} \rightarrow \mathrm{D}} / \mathrm{A}_{\mathrm{D}-\mathrm{W}}$. In 200 table, the properties with the notations, $\mathrm{W}->\mathrm{W}, \mathrm{W}->\mathrm{D}, \mathrm{D}->\mathrm{W}$ and $\mathrm{D}->\mathrm{D}$ mean the values for the hydrogen bond 201 switchings of water from water to water, water to DMSO, DMSO to water and DMSO to DMSO. The error of data refers to the root mean square deviation (RMSD) of the block ensemble averages. 


\begin{tabular}{|c|c|c|c|c|c|c|c|c|c|c|}
\hline$x_{\mathrm{D}}$ & 0 & 0.05 & 0.15 & 0.25 & 0.35 & 0.50 & 0.66 & 0.81 & 0.90 & 1.00 \\
\hline \multicolumn{11}{|c|}{ Diffusion constants, D (unit: $10^{-5} \mathrm{~cm}^{2} / \mathrm{s}$ ) } \\
\hline Water & & $1.63 \pm 0.05$ & $0.89 \pm 0.02$ & $0.33 \pm 0.02$ & $0.21 \pm 0.02$ & $0.18 \pm 0.02$ & $0.31 \pm 0.03$ & $0.51 \pm 0.03$ & $0.74 \pm 0.03$ & \\
\hline DMSO & & $0.77 \pm 0.03$ & $0.38 \pm 0.02$ & $0.23 \pm 0.01$ & $0.17 \pm 0.02$ & $0.21 \pm 0.02$ & $0.42 \pm 0.02$ & $0.75 \pm 0.02$ & $1.21 \pm 0.02$ & $1.56 \pm 0.02$ \\
\hline
\end{tabular}

Total reorientation times of water along $\mathrm{OH}$ bond vector and DMSO along the $\mathrm{O}=\mathrm{S}$ bond vector by fitting with Eq. S2 (ps)

$\begin{array}{lllllllllll}\tau_{2}(\mathrm{OH}) & 2.38 \pm 0.2 & 3.98 \pm 0.2 & 8.34 \pm 0.2 & 16.53 \pm 1.0 & 25.01 \pm 1.1 & 28.66 \pm 2.1 & 19.21 \pm 1.2 & 13.80 \pm 2.0 & 10.18 \pm 1.0 & \\ & & & & & & & & & & \\ \tau_{2}(\mathrm{O}-\mathrm{S}) & & 7.77 \pm 1.5 & 13.69 \pm 0.8 & 20.56 \pm 1.2 & 25.78 \pm 1.0 & 21.16 \pm 0.8 & 14.33 \pm 0.5 & 8.47 \pm 0.5 & 5.33 \pm 0.5 & 3.37 \pm 0.5\end{array}$

Total reorientation times and the total reorientation time of water for each hydrogen bond switching by EMJ model with Eq. S3-S8 (ps)

\begin{tabular}{|c|c|c|c|c|c|c|c|c|c|}
\hline$\tau_{\mathrm{EMJ}}$ & $2.24 \pm 0.2$ & $4.11 \pm 0.2$ & $10.08 \pm 0.2$ & $15.73 \pm 0.6$ & $23.48 \pm 0.6$ & $27.67 \pm 1.0$ & $19.84 \pm 0.4$ & $14.35 \pm 0.4$ & $10.11 \pm 0.5$ \\
\hline$\tau_{\mathrm{EMJ}}^{\mathrm{W}-}>W$ & & $3.9 \pm 0.23$ & $9.10 \pm 0.2$ & $14.46 \pm 0.8$ & $22.53 \pm 0.8$ & $30.91 \pm 1.9$ & $25.68 \pm 1.1$ & $20.88 \pm 1.1$ & $17.97 \pm 1.5$ \\
\hline$\tau_{\mathrm{EMJ}}^{\mathrm{W}->D}$ & & $4.81 \pm 0.45$ & $11.33 \pm 0.2$ & $17.04 \pm 0.8$ & $26.3 \pm 1.1$ & $33.46 \pm 1.9$ & $24.22 \pm 0.9$ & $17.36 \pm 0.9$ & $13.68 \pm 0.9$ \\
\hline$\tau_{\mathrm{EMJ}}^{\mathrm{D}}>W$ & & $6.11 \pm 0.81$ & $12.12 \pm 0.8$ & $18.19 \pm 1.2$ & $23.53 \pm 1.3$ & $24.65 \pm 1.8$ & $18.54 \pm 0.7$ & $14.89 \pm 0.5$ & $11.54 \pm 0.5$ \\
\hline$\tau_{\mathrm{EMJ}}^{\mathrm{D}->D}$ & & $7.47 \pm 0.87$ & $11.63 \pm 0.9$ & $16.78 \pm 1.6$ & $21.19 \pm 1.4$ & $23 \pm 2.2$ & $16.89 \pm 0.8$ & $12.55 \pm 0.6$ & $9.25 \pm 0.6$ \\
\hline
\end{tabular}

Weighted factors of Eq. S4 for each type of hydrogen bond switching

\begin{tabular}{|c|c|c|c|c|c|c|c|c|c|c|}
\hline $\mathrm{A}_{\mathrm{W}->\mathrm{W}}$ & 1.00 & $0.91 \pm 0.01$ & $0.68 \pm 0.01$ & $0.55 \pm 0.01$ & $0.41 \pm 0.01$ & $0.20 \pm 0.01$ & $0.08 \pm 0.01$ & $0.03 \pm 0.01$ & $0.00 \pm 0.01$ & \\
\hline$A_{D->D}$ & & $0.00 \pm 0.01$ & $0.03 \pm 0.01$ & $0.04 \pm 0.01$ & $0.09 \pm 0.01$ & $0.18 \pm 0.01$ & $0.29 \pm 0.01$ & $0.54 \pm 0.01$ & $0.72 \pm 0.01$ & 1.00 \\
\hline$\tau_{\mathrm{HB}}^{\mathrm{W}}->W$ & 3.26 & $4.77 \pm 0.1$ & $11.76 \pm 0.3$ & $15.98 \pm 1.1$ & $25.68 \pm 2.0$ & $45.15 \pm 3.5$ & $58.62 \pm 3.1$ & $61.81 \pm 4.2$ & $53.99 \pm 5.9$ & \\
\hline$\tau_{\mathrm{HB}}^{\mathrm{W}->D}$ & & $5.98 \pm 0.1$ & $12.39 \pm 0.3$ & $19.47 \pm 1.0$ & $31.29 \pm 2.5$ & $52.70 \pm 3.0$ & $61.90 \pm 3.5$ & $68.56 \pm 4.3$ & $74.14 \pm 5.6$ & \\
\hline$\tau_{\mathrm{HB}}^{\mathrm{D}}->W$ & & $9.00 \pm 0.2$ & $19.10 \pm 0.5$ & $26.94 \pm 2.3$ & $38.71 \pm 3.2$ & $58.47 \pm 3.2$ & $84.88 \pm 3.8$ & $101.56 \pm 4$ & $114.76 \pm 6$ & \\
\hline & & 0.66 & 0.67 & 0.72 & 0.81 & 0.90 & 0.73 & 0.64 & 0.65 & \\
\hline \multicolumn{11}{|c|}{ Equilibration constants, $\mathrm{K}=\mathrm{A}_{\mathrm{W}->\mathrm{D}} / \mathrm{A}_{\mathrm{D}->\mathrm{W}}$} \\
\hline & & 0.67 & 0.65 & 0.60 & 0.67 & 0.82 & 0.80 & 0.57 & 0.55 & \\
\hline \multicolumn{11}{|c|}{ Average jump angle of each hydrogen bond switching, $\theta^{i->j}$ (degree) } \\
\hline$\theta^{\mathrm{W}->W}$ & & $68.23 \pm 2.0$ & $64.71 \pm 1.7$ & $60.41 \pm 1.5$ & $56.93 \pm 1.5$ & $53.98 \pm 1.0$ & $54.80 \pm 1.0$ & $55.99 \pm 1.5$ & $53.63 \pm 1.5$ & \\
\hline
\end{tabular}




\begin{tabular}{|c|c|c|c|c|c|c|c|c|c|}
\hline$\theta^{\mathrm{W}} \rightarrow>D$ & & $70.92 \pm 2.6$ & $63.89 \pm 2.1$ & $62.52 \pm 1.5$ & $58.81 \pm 1.5$ & $54.37 \pm 1.0$ & $50.98 \pm 1.0$ & $51.37 \pm 1.0$ & $50.08 \pm 1.2$ \\
\hline$\theta^{\mathrm{D}->W}$ & & $71.27 \pm 3.2$ & $64.52 \pm 1.5$ & $62.99 \pm 1.5$ & $58.98 \pm 1.5$ & $54.93 \pm 1.0$ & $50.81 \pm 1.0$ & $52.06 \pm 1.0$ & $49.39 \pm 1.0$ \\
\hline$\theta^{\mathrm{D}->D}$ & & $81.25 \pm 3.0$ & $82.84 \pm 2.0$ & $83.44 \pm 2.0$ & $82.38 \pm 2.6$ & $82.54 \pm 2.8$ & $82.92 \pm 2.7$ & $83.20 \pm 2.5$ & $84.32 \pm 3.1$ \\
\hline \multicolumn{10}{|c|}{ Jump reorientation time of water, $\tau_{j}^{\mathrm{j}->j}$ for each type of hydrogen bond switching (ps) } \\
\hline$\tau_{\jmath}^{W->W}$ & $3.50 \pm 0.2$ & $6.18 \pm 0.1$ & $15.33 \pm 0.2$ & $23.85 \pm 1.1$ & $40.65 \pm 1.0$ & $75.72 \pm 3.5$ & $100.56 \pm 3$ & $103.20 \pm 4$ & $97.94 \pm 6$ \\
\hline$\tau_{\mathrm{J}}^{\mathrm{W} \rightarrow D}$ & & $7.68 \pm 0.1$ & $18.78 \pm 0.2$ & $29.52 \pm 1.1$ & $51.29 \pm 1.5$ & $93.52 \pm 3.0$ & $114.57 \pm 3$ & $129.85 \pm 4$ & $149.75 \pm 6$ \\
\hline$\tau_{j}^{\mathrm{D}->W}$ & & $11.57 \pm 0.2$ & $26.47 \pm 0.5$ & $40.71 \pm 2.1$ & $62.98 \pm 2.2$ & $103.92 \pm 2$ & $158.44 \pm 4$ & $192.47 \pm 4$ & $222.31 \pm 6$ \\
\hline$\tau_{J}^{\mathrm{D}} \rightarrow>D$ & & $14.68 \pm 0.5$ & $22.98 \pm 0.8$ & $32.18 \pm 2.6$ & $46.52 \pm 2.1$ & $78.04 \pm 3.8$ & $119.34 \pm 4$ & $151.30 \pm 5$ & $163.57 \pm 6$ \\
\hline \multicolumn{10}{|c|}{ Frame reorientation time of water, $\tau_{f}^{i->j}$ for each type of hydrogen bond switching (ps) } \\
\hline$\tau_{\mathrm{f}}^{W->W}$ & $6.25 \pm 0.2$ & $10.59 \pm 0.5$ & $22.37 \pm 0.2$ & $36.70 \pm 0.5$ & $50.56 \pm 1.0$ & $52.22 \pm 1.5$ & $34.48 \pm 0.7$ & $26.18 \pm 0.5$ & $22.01 \pm 0.5$ \\
\hline$\tau_{\mathrm{f}}^{\mathrm{W}->D}$ & & $12.87 \pm 1.0$ & $28.55 \pm 0.2$ & $40.28 \pm 0.7$ & $53.99 \pm 1.3$ & $52.09 \pm 2.0$ & $30.72 \pm 0.5$ & $20.04 \pm 0.6$ & $15.06 \pm 0.5$ \\
\hline$\tau_{\mathrm{f}}^{\mathrm{D}->W}$ & & $12.93 \pm 1.5$ & $22.37 \pm 1.3$ & $32.87 \pm 0.8$ & $37.57 \pm 1.3$ & $32.32 \pm 2.1$ & $21.00 \pm 0.4$ & $16.14 \pm 0.3$ & $12.17 \pm 0.3$ \\
\hline$\tau_{\mathrm{f}}^{\mathrm{D}->D}$ & & $15.23 \pm 1.5$ & $23.56 \pm 1.3$ & $35.08 \pm 0.9$ & $38.91 \pm 1.5$ & $32.62 \pm 2.3$ & $19.68 \pm 0.2$ & $13.68 \pm 0.3$ & $9.81 \pm 0.3$ \\
\hline \multicolumn{10}{|c|}{ Reorientation time of DMSO with the number of hydrogen bond, $\mathrm{n}(\mathrm{n}=0,1$ and 2$)$ at $\mathrm{t}=0(\mathrm{ps})$ along O-S bond vector } \\
\hline$\tau_{2}(\mathrm{O}-\mathrm{S})(\mathrm{n}=0)$ & & $6.72 \pm 2.0$ & $9.38 \pm 1.8$ & $12.63 \pm 1.6$ & $16.36 \pm 1.2$ & $12.03 \pm 0.6$ & $8.73 \pm 0.5$ & $5.92 \pm 0.5$ & $3.99 \pm 0.5$ \\
\hline$\tau_{2}(\mathrm{O}-\mathrm{S})(\mathrm{n}=1)$ & & $7.94 \pm 1.8$ & $13.19 \pm 0.8$ & $19.13 \pm 1.3$ & $24.29 \pm 1.0$ & $21.61 \pm 0.8$ & $16.77 \pm 0.5$ & $12.27 \pm 0.4$ & $9.27 \pm 0.5$ \\
\hline$\tau_{2}(\mathrm{O}-\mathrm{S})(\mathrm{n}=2)$ & & $7.93 \pm 1.5$ & $14.90 \pm 0.8$ & $21.34 \pm 1.2$ & $27.69 \pm 1.1$ & $24.19 \pm 1.2$ & $17.39 \pm 0.5$ & $12.42 \pm 0.7$ & $9.90 \pm 0.8$ \\
\hline
\end{tabular}

205 References:

206 1. B. Bagchi, "Water Dynamics in the Hydration Layer around Proteins and Micelles," Chem. Rev. $207 \quad 105,3197$ (2005).

208 2. (a) D. Laage and J. T. Hynes, "Reorientation and jump mechanism of water," Science 311, 832 209 (2006). (b) D. Laage and J. T. Hynes, “On the molecular mechanism of water reorientation,” J. Phys. Chem B 112, 14230-14242 (2008). (c) D. Laage, G. Stirnemann, and J. T. Hynes, "Why Water Reorientation Slows without Iceberg Formation around Hydrophobic Solutes?" J. Phys. Chem. B $113,2428-2435(2009)$.

3. Q. Zhang, T. Wu, C. Chen, S. Mukamel and W. Zhuang, "Molecular mechanism of water reorientational slowing down in concentrated ionic solutions," Proc. Natl. Acad. Sci. U S A. 114, 10023-10028 (2017). 
216 4. Q. Zhang, H. Chen, T. Wu, T. Jin, R. Zhang, Z. Pan, J. Zheng, Y. Gao and W. Zhuang, "The 217 opposite effects of sodium and potassium cations on water dynamics," Chem. Sci. 8, 1429-1435 $218 \quad$ (2017).

219 5. G. Stirnemann, E. Wernersson, P. Jungwirth and D. Laage, "Mechanisms of acceleration and retardation of water dynamics by ions," J. Am. Chem. Soc. 135, 11824-11831 (2013).

221 6. E. N. Ivanov, “Theory of Rotational Brownian Motion,” Sov. Phys. JETP 18, 1041-1045 (1964).

222 7. D. Laage, T. Elsaesser and J. T. Hynes, "Water Dynamics in the Hydration Shells of Biomolecules," $223 \quad$ Chem. Rev. 117, 10694 (2017).

224 8. J. F. Kincaid, H. Eyring and A. E. Stearn, "Viscosity and diffusion in the liquid state," Chem. Rev. $225 \quad 28,301-65(1941)$.

226 9. (a) J. Qvist, H.Schober and B. Halle, "Structural dynamics of supercooled water from quasielastic neutron scattering and molecular simulations,” J. Chem. Phys. 134, 144508 (2011). (b) J. Qvist, C. Mattea, E. P. Sunde and B. Halle, "Rotational dynamics in supercooled water from nuclear spin relaxation and molecular simulations," J. Chem. Phys. 136, 204505 (2012). 